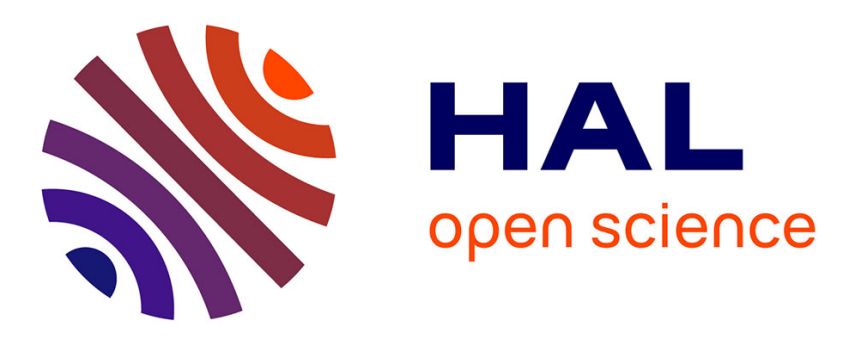

\title{
A Traffic Camera Calibration Method Based on Multi-rectangle
}

Liying Lu, Xiaobo Lu, Saiping Ji, Chen Tong

\section{To cite this version:}

Liying Lu, Xiaobo Lu, Saiping Ji, Chen Tong. A Traffic Camera Calibration Method Based on Multi-rectangle. 8th International Conference on Intelligent Information Processing (IIP), Oct 2014, Hangzhou, China. pp.230-238, 10.1007/978-3-662-44980-6_26 . hal-01383337

\section{HAL Id: hal-01383337 \\ https://inria.hal.science/hal-01383337}

Submitted on 18 Oct 2016

HAL is a multi-disciplinary open access archive for the deposit and dissemination of scientific research documents, whether they are published or not. The documents may come from teaching and research institutions in France or abroad, or from public or private research centers.
L'archive ouverte pluridisciplinaire HAL, est destinée au dépôt et à la diffusion de documents scientifiques de niveau recherche, publiés ou non, émanant des établissements d'enseignement et de recherche français ou étrangers, des laboratoires publics ou privés.

\section{(c)(1)}

Distributed under a Creative Commons Attribution| 4.0 International License 


\title{
A Traffic Camera Calibration Method Based On Multi-Rectangle
}

\section{Calibrating a camera using multi-rectangle constructed by mark lines in traffic road}

\author{
Liying Lu, Xiaobo Lu*, Saiping Ji, Chen Tong \\ Key Laboratory of Measurement and Control of CSE \\ Ministry of Education \\ School of Automation, Southeast University \\ Nanjing, 210096, China \\ 8618994100907 \\ *E-mail:xblu2013@126.com
}

\begin{abstract}
As distance detection is frequently needed in modern traffic management, camera calibration has a significant influence on the accuracy of distance detection. The traditional calibration methods usually need a calibration target, and the exact distance of calibration points has to be measured. In this paper, according to the camera imaging model, we put forward a calibration method based on multi-rectangle, which constructs several rectangles with the mark lines of the traffic road. Without measuring the internal or external parameters of the camera or the exact distance of calibration point, we only need the side lengths of rectangles and the image coordinates of the rectangular vertexes to establish the video image distance conversion model. For improving the accuracy of conversion, we use multiple rectangles to determine the coordinates of the vanishing points and modify the coordinates of the rectangular vertexes. Experimental results show that our method is more accurate when compared with the mainstream methods.
\end{abstract}

KEYWORDS: distance detection; camera calibration; multi-rectangle; parameter modification 


\section{Introduction}

In modern traffic management, traffic video image processing is frequently used in the field of distance detection, speed detection and visibility detection. As an important part of traffic image processing, camera calibration has a significant influence on the accuracy of detections which related to distance. Camera calibration means establishing the conversion model between the position of image pixel point and the position of actual point. According to the camera model, we get the camera parameters using some feature points of a real scene and these points' coordinates in the image. Finally, we build the conversion model between the image coordinates and the actual distance using the camera parameters (Zheng, 1998; Hartley, 2003).

Traditional camera calibration methods usually need some standard calibration targets, whose actual size has been accurately measured before calibrating. These methods use the corresponding relationship of image points and actual points to establish the constraint conditions of camera parameters, then calculate the parameters of camera by some nonlinear optimization algorithm(Zhang, 2000; Zhang, 2004; Ye). It is been proven that the calibration method based on vanishing point is effective and reliable, the position of vanishing point could be determined by one-dimensional target of calibration targets(Guillou, 2000; Yan, 2013). Wei Geng and Yang Liu put forward a new algorithm based on single rectangle, which uses the mark lines in traffic road to calibrate the parameters of camera, then establish mutual conversion model between image coordinate system and the actual distance(Geng, 2012). The method uses the actual lengths of rectangular patterns and the corresponding information of coordinates to establish detection model, without taking manual measurement to get camera internal and external parameters and the absolute distance of standard fixed points.

But under actual situation, as the mark lines may have been worn and the feature points are manual marked, that method may bring serious error. To solve this problem, a calibration algorith mbased on multi-rectangle is introduced in this paper, which constructs several rectangles with mark lines of the traffic road, then modif ies the Y-axis direction vanishing point by fitting a straight line with mu ltiple points. In the $\mathrm{X}$-axis direction, $\mathrm{n}$ straight lines will generate intersection points, we choose the point with highest accuracy as the $X$-axis direction vanishing point. To get mo re accurate calibration parameters, we use the position information of vanishing points and the accuracy of each rectangular vertex to modify the position of every rectangular vertex, then establish distance conversion model with these modified parameters. Through a lot of experiment, this method is more accurate than the single-rectangle algorithm. 


\section{Calibration Method Based On Multi-Rectangle}

As the white mark lines and the gray road surface make the contrast ratio of the image not strong enough, and the mark lines may have been worn, it' $s$ difficult to localize the position of feature points accurately and the calibration method based on single-rectangle is inclined to bring error. We study a parameter modification method in this paper to reduce the error. As shown in Figure 1, we can construct multiple rectangles in a road surface image.

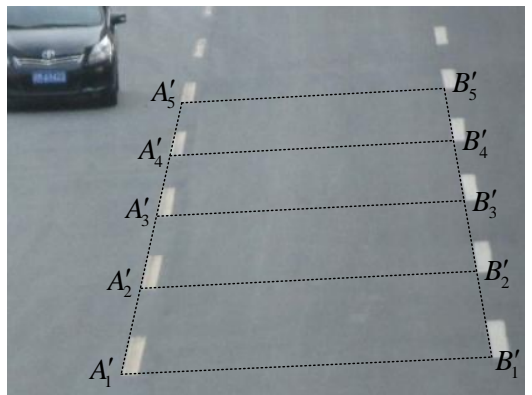

Figure 1. Construct multiple rectang les in a road surface image

\subsection{Modifying the coordinate of the vanishing point in Y-axis direction}

The projection line of mark lines in image plane will intersect at point $Q_{2}^{\prime}$, which is the vanishing point in Y-axis direction. Assume that we construct single rectangle with point $A_{1}^{\prime} A_{2}^{\prime} B_{1}^{\prime} B_{2}^{\prime}$, straight line $A_{1}^{\prime} A_{2}^{\prime}$ and $B_{1}^{\prime} B_{2}^{\prime}$ can be viewed as the projection lines of mark lines, but error will be brought when any point is localized inaccurately. To reduce the error, we fit straight line $L_{A}^{\prime}$ with point $A_{1}^{\prime} A_{2}^{\prime} A_{3}^{\prime} \cdots$ using the least squares method, hence, $L_{A}^{\prime}$ can be viewed as the projection line in image plane of the left mark line. Then we fit the projection line in image plane of the right mark line $L_{B}^{\prime}$ in the same way. Find the intersection point $Q_{2}^{\prime}$ of straight line $L_{A}^{\prime}$ and $L_{B}^{\prime}$, so $Q_{2}^{\prime}$ is the vanishing point in Y-axis direction.

\subsection{Modifying the coordinate of the vanishing point in $X$-axis direction}

Straight line $A_{1} B_{1}, A_{2} B_{2} \cdots A_{n} B_{n}$ in road surface plane all parallel to $\mathrm{X}$-axis, so straight line $A_{1}^{\prime} B_{1}^{\prime}, A_{2}^{\prime} B_{2}^{\prime} \cdots A_{n}^{\prime} B_{n}^{\prime}$ in image plane should intersect at vanishing point $Q_{1}^{\prime}$, but there may be multiple intersection points in actual image, generally, $\mathrm{n}$ straight lines will generate $C_{n}^{2}$ intersection points. Assume that straight line $A_{i}^{\prime} B_{i}^{\prime}$ and $A_{j}^{\prime} B_{j}^{\prime}$ intersect at point $P_{i j}^{\prime}$, the closer $P_{i j}^{\prime}$ near to other straight lines, the closer $P_{i j}^{\prime}$ to the actual position of vanishing point $Q_{1}^{\prime}$. Define $D_{i j}$ as the accuracy of $P_{i j}^{\prime}$ : 


$$
D_{i j}=\left\{\begin{array}{l}
\sum_{k=1}^{n} d_{i j-k}, i \neq j \\
0, i=j
\end{array}\right.
$$

\subsection{Modifying the coordinates of the rectangular vertex}

Some feature points may have been inaccurately localized as we use mark lines to construct calibration target, so these coordinates must be modified.

To characterize the accuracy of point $A_{i}^{\prime}$ and $B_{i}^{\prime}$, we sumall $D_{i k}(1 \leq k \leq n)$ value from point $P_{i 1}^{\prime}$ to point $P_{i n}^{\prime}$ on straight line $A_{i}^{\prime} B_{i}^{\prime}$, then calculate the normalized summation. Define the accuracy of any rectangular vertex in the $Q_{1}^{\prime}$ direction:

$$
E_{s k q 1}=\frac{\sum_{t=1}^{n} D_{k t}}{\max \left(\sum_{t=1}^{n} D_{k t}\right)}
$$

In the formula, $s$ could be $a$ or $b, 1 \leq k \leq n, 1 \leq t \leq n$, so $s k$ represents a rectangular vertex, $\max \left(\sum_{t=1}^{n} D_{k t}\right)$ is the largest value of accuracies on straight line $A_{i}^{\prime} B_{i}^{\prime}, q 1$ means the current value is the accuracy in $Q_{1}^{\prime}$ direction. But $E_{a k q 1}$ equals $E_{b k q 1}$ in $Q_{1}^{\prime}$ direction, which means it's unable to distinguish the accuracy of $A_{i}^{\prime}$ and $B_{i}^{\prime}$. So we have to find other straight lines in other direction, make $A_{i}^{\prime}$ and $B_{i}^{\prime}$ belongs to different straight lines, thus to separate the accuracy of $A_{i}^{\prime}$ and $B_{i}^{\prime}$.

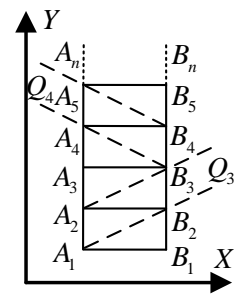

(a)

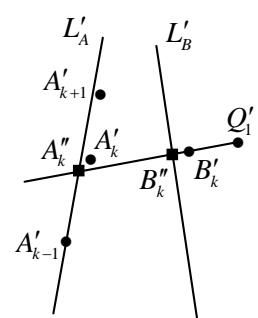

(b)

Figure 2. Feature point coordinate modification

As Figure 2(a) shows, every diagonal in the same direction parallels to each other, as an example, we choose a group of straight lines $A_{i}^{\prime} B_{i+1}^{\prime}(1 \leq i \leq n-1)$, these straight lines will intersect at a vanishing point $Q_{3}^{\prime}$. The coordinate of $Q_{3}^{\prime}$ could be calculated in the same way as how we calculate the coordinate of $Q_{1}^{\prime}$, then the 
accuracy of $A_{i}^{\prime}$ and $B_{i+1}^{\prime}$ in $Q_{3}^{\prime}$ direction $E_{s k q 3}$ can be calculated. As an exception, neither $A_{1}^{\prime}$ nor $B_{n}^{\prime}$ belongs to a straight line in $Q_{3}^{\prime}$ direction, so their $E_{s k q 3}$ value is 0 Similarly, a group of straight lines $A_{i+1}^{\prime} B_{i}^{\prime}(1 \leq i \leq n-1)$ will intersect at vanishing point $Q_{4}^{\prime}$, we get calculate the coordinate of $Q_{4}^{\prime}$ and the $E_{s k q 4}$ value of every feature point. Define the accuracy of a feature point as:

$$
E_{s k}=\frac{E_{s k q 1}+E_{s k q 3}+E_{s k q 4}}{1+e f c\left(E_{s k q 3}\right)+e f c\left(E_{s k q 4}\right)}
$$

In the formula, $k$ ranges from 1 to $n$, efc $\left(E_{s k q 3}\right)=0$ when $s=a, k=n$ or $s=b, k=1 \quad ; \quad e f c\left(E_{s k q^{4}}\right)=0 \quad$ when $\quad s=a, k=1 \quad$ or $\quad s=b, k=n$, otherwise $\operatorname{efc}\left(E_{s k q 3}\right)=1, e f c\left(E_{s k q 4}\right)=1$.

After getting the accuracy of every feature point, we can modify the coordinate of feature points. We take point $A_{k}^{\prime}$ and $B_{k}^{\prime}$ as an example, the exact feature point $A_{k}^{\prime \prime}, B_{k}^{\prime \prime}$ should be the intersection point of the straight lines which pass through $Q_{1}^{\prime}$ and straight line $L_{A}^{\prime}$ or $L_{B}^{\prime}$, we mark them as square points in Figure 2(b). The round dots in Figure 2(b) represents the manual marked feature points $A_{k}^{\prime}, B_{k}^{\prime}$. As the accuracy of $A_{k}^{\prime}$ and $B_{k}^{\prime}$ is known, we choose the point which has a smaller accuracy value as reference point, then connect it and $Q_{1}^{\prime}$ to construct the modified straight line $A_{k}^{\prime \prime} B_{k}^{\prime \prime}$, so the intersection point of $A_{k}^{\prime \prime} B_{k}^{\prime \prime}$ and $L_{A}^{\prime}$ is the modified point $A_{k}^{\prime}$, the intersection point of $A_{k}^{\prime \prime} B_{k}^{\prime \prime}$ and $L_{B}^{\prime}$ is the modified point $B_{k}^{\prime}$.

\subsection{Distance conversion model}

Compared to the single-rectangle method, the multi-rectangle method make $Q_{1}^{\prime}$, $Q_{2}^{\prime}$ and every rectangular vertexes modified, so we can get more accurate calibration parameters.

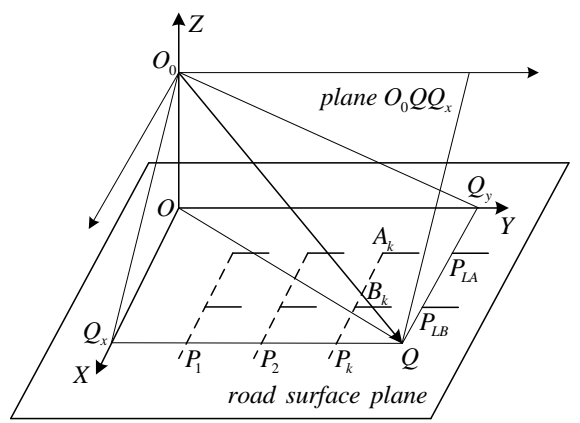

Figure 3. Calculating calibration parameters 
According to camera imaging model, we can calculate scale factor $k_{11}$ and $k_{12}$ in X-axis direction. As Figure 3 shows, $P_{L A}$ is the intersect point of mark line $L_{A}$ and plane $O_{0} Q Q_{y}, P_{L B}$ is the intersection point of mark line $L_{B}$ and plane $O_{0} Q Q_{y}$. As $Q Q_{y}$ parallels to $\mathrm{X}$-axis, its projection line in image plane passes through $Q_{1}^{\prime}$, so $Q_{1}^{\prime} Q^{\prime}$ is the projection line of $Q Q_{y}, P_{L A}^{\prime}$ is the projection point of $P_{L A}$, and it is the intersection point of $Q_{1}^{\prime} Q^{\prime}$ and $L_{A}^{\prime}, P_{L B}^{\prime}$ is the projection point of $P_{L B}$, and it is the intersection point of $Q_{1}^{\prime} Q^{\prime}$ and $L_{B}^{\prime}$, let $l_{x a}$ represents the X-axis coordinate of $P_{L A}$, $l_{x b}$ represents the $\mathrm{X}$-axis coordinate of $P_{L B}$, according to the one-dimensional distance conversion model:

$$
\left\{\begin{array}{l}
l_{x a}=k_{11}+k_{12} p_{1} \\
l_{x b}=k_{11}+k_{12} p_{2}
\end{array}\right.
$$

where $p_{1}$ is the reciprocal of the pixel-value differencing between point $P_{L A}^{\prime}$ and $Q_{1}^{\prime}$, $p_{2}$ is the reciprocal of the pixel-value differencing between $P_{L B}^{\prime}$ and $Q_{1}^{\prime}$.

Let $s_{A B}$ represents the distance between $L_{A}$ and $L_{B}, s_{A B}=l_{x b}-l_{x a}=k_{12}\left(p_{1}-p_{2}\right)$, according to highway construction specifications ${ }^{[6]}, s_{A B}=3.75 \mathrm{~m}$, so:

$$
k_{12}=\frac{s_{A B}}{p_{1}-p_{2}}=\frac{3.75}{p_{1}-p_{2}}
$$

According to the relationship between $k_{11}$ and $k_{12}$, we can get $k_{11}$ :

$$
k_{11}=-\frac{k_{12} \sin \alpha_{1} \cos \alpha_{1}}{f_{d}}
$$

Then we calculate scale factor $k_{21}$ and $k_{22}$ in $\mathrm{Y}$-axis direction. As rectangular vertexes have been modified, every straight line $A_{k}^{\prime} B_{k}^{\prime}$ passes through $Q_{1}^{\prime}$, so straight line $A_{k} B_{k}$ parallels to $\mathrm{X}$-axis, assume the intersection point of $A_{k} B_{k}$ and plane $O_{0} Q Q_{x}$ is $P_{k}(1 \leq k \leq n)$, the actual distance between every two points is easily calculated according to highway construction specifications. $P_{k}^{\prime}$ is the projection point of $P_{k}$, and it is also the intersection point of straight line $A_{k}^{\prime} B_{k}^{\prime}$ and $Q_{2}^{\prime} Q^{\prime}$. According to the one-dimensional distance conversion model in $\mathrm{Y}$-axis direction, for point $P_{1}^{\prime}: l_{y 1}=k_{21}+k_{22} p_{21}$, where $l_{y 1}$ is the Y-axis coordinate of $P_{1}^{\prime}$, $p_{21}$ is the reciprocal of the pixel-value differencing between point $P_{1}^{\prime}$ and $Q_{2}^{\prime}$, which could be easily calculated in image. Similarly, for $P_{k}^{\prime}: l_{y 1}+d_{k}=k_{21}+k_{22} p_{2 k}$, where $d_{k}$ represents the distance between $P_{k}$ and $P_{1}, p_{2 k}$ represents the reciprocal of the pixel-value differencing between point $P_{k}^{\prime}$ and $Q_{2}^{\prime}$. As $k$ ranges from 1 to $n$, we can get $n$ equations, then we can establish a coordinate system with 
$\left(l_{y 1}+d_{k}\right)$-axis as vertical axis and $p_{2 k}$-axis as horizontal axis, fit a straight line using the points we got in the equations, the slope of the straight line is $k_{22}$. According to the relationship between $k_{21}$ and $k_{22}: k_{21}=-k_{2} \sin \alpha_{1} \cos \alpha_{1} / f_{d}$. In conclusion, we can establish the two-dimensional distance conversion model as:

$$
\left\{\begin{array}{l}
l_{x}=k_{12} p_{1}-k_{12} \sin \alpha_{1} \cos \alpha_{1} / f_{d} \\
p_{1}=1 /\left(\left(u_{1}-u_{x}\right)^{2}+\left(v_{1}-v_{x}\right)^{2}\right)^{1 / 2} \\
l_{y}=k_{22} p_{2}-k_{22} \sin \alpha_{2} \cos \alpha_{2} / f_{d} \\
p_{2}=1 /\left(\left(u_{2}-u_{y}\right)^{2}+\left(v_{2}-v_{y}\right)^{2}\right)^{1 / 2}
\end{array}\right.
$$

\section{Results And Analysis Of Experiment}

\subsection{Accuracy testing experiment}

To evaluate the accuracy of the proposed method, we compare the effect of multi-rectangle method and the single-rectangle method through experiment. We mark six points in image that are corresponding to $21.00 \mathrm{~m}$ to $214.13 \mathrm{~m}$ in actual road plane, then calculate the distance of these points using the video image distance conversion model, and compare the results with the actual distance. As Table 1 shows, compared to the single-rectangle method, the proposed method has an improved measuring accuracy on each distance, and as the distance increases, the error of single-rectangle method increases fast, but the error of the proposed method remains in the range of $5 \%$. In the study we found that the accuracy of vanishing point $Q_{1}^{\prime}$ and $Q_{2}^{\prime}$ have a major impact on the model parameters, for the proposed method, we use multiple rectangles to determine the position of vanishing point, so we could get more accurate vanishing point coordinate than the single-rectangle method.

\begin{tabular}{|c|c|c|c|c|}
\hline \multirow{2}{*}{$\begin{array}{c}\text { Actual distance } \\
(\mathrm{m})\end{array}$} & \multicolumn{2}{|c|}{ Single-rectangle method } & \multicolumn{2}{c|}{ Multi-rectangle method } \\
\cline { 2 - 5 } & $\begin{array}{c}\text { Test value } \\
(\mathrm{m})\end{array}$ & $\begin{array}{c}\text { relative } \\
\text { error }\end{array}$ & $\begin{array}{c}\text { Test value } \\
(\mathrm{m})\end{array}$ & $\begin{array}{c}\text { relative } \\
\text { error }\end{array}$ \\
\hline 21.00 & 20.25 & $3.55 \%$ & 20.52 & $2.30 \%$ \\
\hline 37.69 & 36.94 & $2.00 \%$ & 36.97 & $1.91 \%$ \\
\hline 62.41 & 59.59 & $4.52 \%$ & 60.91 & $2.40 \%$ \\
\hline 104.08 & 97.60 & $6.23 \%$ & 100.82 & $3.13 \%$ \\
\hline 160.47 & 147.83 & $7.88 \%$ & 154.79 & $3.54 \%$ \\
\hline 214.13 & 193.56 & $9.61 \%$ & 205.52 & $4.02 \%$ \\
\hline
\end{tabular}

Table 1. Results for distance detection experiment 


\subsection{Stability testing experiment}

To evaluate the stability of the proposed method, we measure the maximum error of the single-rectangle method and the multi-rectangle method in 10 different traffic images, the distance of each test point is less than 200 meters.

\begin{tabular}{|c|c|c|}
\hline $\begin{array}{c}\text { serial } \\
\text { number }\end{array}$ & $\begin{array}{c}\text { Maximum error (single- } \\
\text { rectangle method) }\end{array}$ & $\begin{array}{c}\text { Maximum error (multi- } \\
\text { rectangle method) }\end{array}$ \\
\hline 1 & $9.61 \%$ & $4.02 \%$ \\
\hline 2 & $12.37 \%$ & $4.72 \%$ \\
\hline 3 & $9.88 \%$ & $5.14 \%$ \\
\hline 4 & $16.24 \%$ & $6.93 \%$ \\
\hline 5 & $8.75 \%$ & $4.12 \%$ \\
\hline 6 & $10.03 \%$ & $5.62 \%$ \\
\hline 7 & $21.72 \%$ & $7.44 \%$ \\
\hline 8 & $10.94 \%$ & $5.92 \%$ \\
\hline 9 & $11.41 \%$ & $5.37 \%$ \\
\hline 10 & $9.18 \%$ & $4.58 \%$ \\
\hline
\end{tabular}

Table 2. Results of stability testing experiment

As Table 2 shows, the maximum error of the multi-rectangle method floats around $5 \%$, while the maximum error of the single-rectangle method floats around $10 \%$. In addition, in the 4 th and 7 th image, the maximum error of the singlerectangle method rise to $16.24 \%$ and $21.72 \%$ due to the mark line has been worn, while the maximum error of the multi-rectangle is only $6.93 \%$ and $7.44 \%$. Through this experiment, we can see the proposed method is more stable than the singlerectangle method.

\section{Conclusion}

This paper put forward a camera calibration algorithm based on multi-rectangle, which constructs multiple rectangles with mark lines in actual traffic road, then modifies the calibration parameters and establish video image distance conversion model. We modify the coordinate of vanishing point in $\mathrm{Y}$-axis direction by fitting a straight line with multiple rectangular vertexes, modify the coordinate of vanishing point in $\mathrm{X}$-axis direction by choosing the point with highest accuracy among all intersection points, then we modify the position of every rectangular vertex, and establish the distance conversion model using the modified parameters. Compared to single-rectangle method, the proposed method provides more accurate calibration parameters, and the distance conversion model we got has an improve accuracy. Experiment result shows that as distance increases, the detecting error increases too, but the effect of the proposed method is still better than the single-rectangle method. 
However, this method still belong to the category of manual calibration, the initial feature points still needs to be manual marked, that would bring some unknown error and reduce the calibration accuracy. How to automatically localize the feature points, that's what we need to improve in further study.

\section{Acknowledgments}

This work was supported by the National Natural Science Foundation of China (No.61374194) and China Postdoctoral Science Foundation Funded Project (No.2013M540405).

\section{References}

China Ministry of Transportation , "Road traffic signs and markings set norms", JTG D822009.

Geng W, "Detection Algorithm of Video Image Distance Based on Rectangular Pattern", 2012 5th International Congress on Image and Signal Processing, 1013-1017.

Gonzales, “Digital Image Processing ”. Beijing: Publishing House of Electronics Industry, 2007.

Guillou, "Using vanishing points for camera calibration and coarse $3 D$ reconstruction from a single image", The Visual Computer, Vol.16, 2000, pp. 396-410.

Hartley et al, "Multiple view geometry in computer vision", Cambridge University Press, 2003.

Hautière et al, "Daytime Visibility Range Monitoring through use of a Roadside Camera", IEEE Intelligent Vehicles Sy mposium, 2008, pp. 470-475.

Yan H, "Camera Self-Calibration In Highway Dynamic Environment", Computer Aided Design and Computer Graphics, Vol. 25, No 7, 2013, pp. 892-899.

Ye Y, "Study on the Camera Calibration Approach and Algorithm of Edge Detection and Contour Tracking”, Dalian :Dalian University of Technology.

Yin W, “Camera calibration based on OpenCV', Computer Engineering and Design, Vol.28, No.1, 2007, pp. 197-199.

Zheng N, “Computer Vision and Pattern Recognition”, Defense Industry Press, Beijing, 1998.

Zhang Z, "A flexible new technique for camera calibration", IEEE transactions on pattern analy sis and machine intelligence, Vol. 22, 2000, pp.1330-1334.

Zhang Z, "Camera calibration with one-dimensional objects", IEEE transactions on pattern analy sis and machine intelligence, Vol. 26, 2004, pp. 892-899. 